\title{
Многозадачность в виртуальной среде: эмпирическое исследование
}

\author{
А.Е. Войскунский, Е.М. Хохлова, О.В. Митина, Е.А. Дорохов \\ МГУ им. М.В. Ломоносова \\ vae-msu@mail.ru, elizabethxoxa@gmail.com, omitina@inbox.ru, \\ dorohov.e@mail.ru
}

\section{Аннотация}

Проблематика многозадачности, т.е. выполнения субъектом сразу нескольких различающихся между собой видов активности, приобрела значительный интерес и в научном сообществе, и у широких масс неспециалистов. Интерес этот объясняется изменением ритма жизни в современную эпоху и, в частности, быстрым развитием электронных цифровых устройств, которые все шире опосредствуют выполняемые людьми виды деятельности. В немалой степени интерес обусловлен многочисленными наблюдениями, согласно которым представители молодежных поколений не только не испытывают проблем в одновременном выполнении различающихся действий, но и явно предпочитают именно такой стиль жизненной активности. В работе ставится цель выявить специфику явления многозадачности в условиях применения системы виртуальной реальности. Исследование проведено в комнате виртуальной реальности типа CAVE на факультете психологии МГУ. Задание для испытуемых состояло в аудировании текстов перед виртуальной аудиторией (учебный класс с 12 учащимися) и одновременном наблюдении за поведением виртуальных аватаров, составляющих аудиторию. Аватары имели фиксированную свободу поведения, при этом их поведение было запрограммировано и было одинаковым для всех испытуемых. Задание для испытуемых (50 студентов разных вузов Москвы) постепенно усложнялось, о степени сложности можно судить по количеству и качеству ошибок, допускаемых испытуемыми в аудировании и в наблюдении за аудиторией. Исследование показало, что затруднения в одновременно реализуемых деятельностях не оказывают значимого влияния друг на друга.

Ключевые слова: многозадачность, медиамногозадачность, виртуальная реальность, внимание, ресурсная модель, аватар, аудирование

Библиографическая ссылка: Войскунский А.Е., Хохлова Е.М., Митина О.В., Дорохов Е.А. Многозадачность в виртуальной среде: эмпирическое исследование // Информационное общество: образование, наука, культура и технологии будущего. Выпуск 4 (Труды XXIII Международной объединенной научной конференции «Интернет и современное общество», IMS-2020 (сборник научных статей). - СПб: Университет ИТМО, 2020. С. 129-142. DOI: 10.17586/2587-8557-2020-4-129-142

\section{Введение}

Явление многозадачности, т.е. выполнения субъектом сразу нескольких различающихся видов активности, оказалась актуальным и для представителей научного сообщества - экономистов, психологов, физиологов, и для менеджеров разного уровня вместе с преподавателями и коучами искусства руководства организациями, и для разработчиков новых образцов оборудования для профессиональной и обыденной жизни, и - не в последнюю очередь - для широких масс пользователей ультрасовременных 
(преимущественно электронных) изделий. Актуальность объясняется убыстрением ритма жизни в современную эпоху, а также быстрым развитием цифровых устройств, которые призваны опосредствовать выполняемые специалистами виды деятельности.

Проблематика многозадачности связывается в психологии с личностными особенностями, с переключаемостью внимания, со стилем жизни представителей молодежных поколений. У многозадачности есть как сторонники, так и противники. Многозадачность часто понимается как межпоколенческий параметр. Для подростков вошло в привычку совмещение нескольких дел, например, прослушивание музыки или просмотр телевизора одновременно с выполнением домашнего задания и ответом на поступившие электронные письма. Широкую известность термин «многозадачность» приобрел в связи с развитием медиа и возрастающим проникновением персонализированных цифровых устройств в повседневную жизнь человека.

\section{1. Феноменология многозадачности}

\section{1. Многозначность с позиций психологии внимания}

Несмотря на распространенное мнение, что в ситуации многозадачности человек способен эффективно выполнить больше заданий, чем если бы занимался ими по очереди, на практике часто оказывается, что продуктивность на самом деле понижается. В литературе представлено достаточно часто высказываемое мнение, согласно которому феномен многозадачности на самом деле отсутствует, а в реальности имеется разве что феномен быстрого переключения между решаемыми задачами [6;9]. Если же он тем не менее реален, как многие полагают, то этот феномен представлен в разных поколениях существенно различающимся образом.

Действительно, согласно распространенному мнению, старшему поколению труднее адаптироваться в современной жизни с ее нарастающими сложностью и скоростью, в то время как когнитивная система детей, родившихся в этой среде, претерпевает определенные изменения в развитии базовых психических процессов [15]. Современные дети, подстраиваясь к условиям постоянно изменяющегося мира, для компенсации ограниченности своих когнитивных и социальных ресурсов вырабатывают комфортные для себя способы защиты от коммуникационных и информационных перегрузок, а также расширяют свои когнитивные возможности при помощи современных технологий [10; 24$]$. Им гораздо легче, чем их отцам и дедам, оперировать большим и даже очень большим количеством параллельно идущих информационных потоков.

Кроме того, современные дети и подростки рано начинают пользоваться различными цифровыми устройствами как для поиска информации, так и для общения и развлечения. Это ложится в основу режима многозадачности, в котором они продолжают пребывать и на протяжении взрослой жизни [18; 24]. Постоянное переключение с одной деятельности на другую не позволяет ребенку надолго зафиксировать внимание на одной активности или одном объекте и погрузиться в суть проблемы [10; 12]. Таким образом, «цифровое поколение» иначе, чем предшествующие поколения, управляет собственными восприятием, вниманием и памятью, мыслительными операциями, социальными процессами $[15 ; 18]$.

Относительно недавно заговорили о новом варианте многозадачности медиамногозадачности. Данный феномен означает параллельное использование технических устройств, переключение между источниками информации, одновременное получение информации из онлайн- и офлайн-источников. Согласно данным американских исследователей, подростки все больше времени проводят в режиме медиамногозадачности $[14 ; 20]$. Все чаще школьники при выполнении домашнего задания вовлечены в различные медиа-активности, например, проводят время в социальных сетях. Согласно данным опроса Common Sense Media, в 2015 году каждый второй подросток сообщил, что он часто 
(21\%) или иногда (29\%) пользуется социальными медиа при выполнении домашнего задания. При этом большинство респондентов не считали, что многофункциональность негативно сказывается на их продуктивности [10;26].

Имеются определенные данные о влиянии пола, возраста и типа культуры на варианты многозадачности. Так, мальчики младшего школьного возраста чаще делают домашнее задание одновременно с игрой в компьютерные игры, а девочки того же возраста - с онлайн-перепиской и прослушиванием музыки. Что касается подростков 13-16 лет, то они чаще совмещают интернет-серфинг с прослушиванием музыки, в то время как взрослые в возрасте 50-65 лет чаще всего одновременно проверяют почту и слушают радио [23]. Кроме того, по данным голландских ученых, характер медиамногозадачности зависит от типа культуры. Так, в полихронных культурах (например, Россия, страны Латинской Америки, Франция, Испания) способность к многозадачности выше, чем в монохронных (например, США, Германия, Англия) [24].

В результате проведенного в 2017 году исследования [10], в котором приняли участие 50 школьников 7-11 лет и их родители, установлено, что $84 \%$ родителей постоянно или часто находятся в режиме многозадачности, однако только $28 \%$ считает, что они успешны в одновременном выполнении нескольких дел, а 70\% признаются, что только часть видов деятельности у них получается выполнить хорошо. Что касается школьников, то среди них только 30\% - «постоянные» или «частые» многозадачники. Однако стоит обратить внимание, что именно в этом возрасте дети начинают приспосабливаться к информационному обществу и адаптироваться в нем, при этом почти каждому третьему из них уже знаком режим медиамногозадачности. Согласно тем же данным, только 14\% детей считают, что они успешные многозадачники, 66\% сообщают, что при выполнении нескольких дел сразу у них есть и успехи, и неудачи, а 14\% считают, что выполнение всех задач им не очень хорошо удается. Стоит отметить и тот факт, что многозадачность взрослых и детей имеет качественные различия: так, родители прибегают к одновременному выполнению нескольких дел в связи с недостатком времени и необходимостью повышения своей эффективности, в то время как для детей режим многозадачности становится привычным форматом выполнения различных задач, поскольку работать становится интереснее и удобнее [10].

В той же работе напоминается, что с позиций нейрофизиологии исследование феномена многофункциональности связано с такой характеристикой человеческого мозга, как нейропластичность. Данный термин был введен в 1930-х гг. учеником И.П. Павлова Е. Конорским. Нейропластичность - это способность мозга изменяться под воздействием опыта и адаптироваться к внешним условиям на протяжении всей жизни. Такая способность позволяет быстро переключаться между задачами. В настоящее время также немало говорится о пластичности головного мозга. Достаточно распространенным является мнение о том, что ребенок должен начать развивать эту способность как можно раньше, дабы достигнуть как можно большего в современном мире, где так поощряется многозадачность [21].

В то же время большое количество исследователей считают, что многозадачность снижает эффективность и качество выполнения различных типов задач. Так, например, если человек одновременно ведет автомобиль и разговаривает по телефону, выполнение и той, и другой задачи становится хуже, чем если бы они выполнялись по отдельности [21]. Это можно объяснить тем, что восприятие речи значительно снижает активность той зоны мозга, которая задействована при вождении автомобиля, что, в свою очередь, приводит к ухудшению бдительности и зрительного внимания. Таким образом, дополнительная задача может ухудшить решение основной задачи.

Несмотря на тот факт, что стиль жизни у представителей цифрового поколения во многом схож, все же можно наблюдать индивидуальные различия по степени многозадачности. На основе проведенных исследований принято разделять многозадачников на «легких» и «тяжелых» в зависимости от количества задач, которые он 
способен выполнять единовременно [10; 19]. Так, в ряде исследований было показано, что подростки и юноши с высоким уровнем медиамногозадачности характеризуются более широким объемом внимания, а также повышенной способностью к переключению внимания. Исходя из этого, можно предположить, что при решении текущей задачи «тяжелые» многозадачники будут в большей степени подвержены влиянию отвлекающей информации [19]. Кроме того, такие особенности внимания «тяжелых» многозадчников могут способствовать развитию мультисенсорной, или многомодальной интеграции, другими словами, сопоставлению информации, которая поступает от разных органов чувств [17]. Среди предикторов высокого уровня многозадачности выделяются такие факторы, как высокие показатели рабочей памяти, креативность, повышенная импульсивность, тяга к новым впечатлениям, а также медиаориентированность семьи [25]. «Легкие» многозадачники эффективнее справляются с решением единственной задачи. При пониженном уровне многозадачности их рабочая память, механизмы переключения внимания, торможения и контроля импульсов работают эффективнее [19].

«Тяжелые» многозадачники хотя они лучше и быстрее справляются с параллельным решением нескольких задач, все же обладают сниженной устойчивостью и концентрацией внимания, а также способны удерживать в рабочей памяти ограниченное количество оперативной информации, необходимой для решения текущей задачи. Кроме того, широкая зона внимания «тяжелых» многозадачников и сниженная производительность рабочей памяти негативно сказываются на объеме долговременной памяти [19].

Установлено, что при повышении уровня многозадачности участники становятся рассеянными и заметно отвлекаются от главной задачи; сделан вывод, что при большом количестве раздражителей, вне зависимости от пола и возраста, человеку трудно отличать важные факторы от маловажных [18]. Австралийские исследователи показали, что из-за широкой зоны внимания и большого количества поступающей информации способность к eе усвоению у «тяжелых» многозадачников снижена. Также для «тяжелых» многозадачников характерен «поверхностный» стиль деятельности, что, в свою очередь, ведет к снижению качества переработки информации, анализа проблемы и к большему числу ошибок [13].

Проанализировав приведенные выше исследования, можно сделать следующие выводы. В ситуации ограниченного количества времени «тяжелые» многозадачники быстрее и успешнее вникают в суть проблемы, решают параллельно несколько задач и рационально распределяют время. В то же время быстрый темп и широкий охват задач препятствует возможности глубокого и детального изучения задачи и способствует формированию поверхностного стиля работы. «Легкие» же многозадачники подробно и качественно анализируют одну конкретную задачу, однако последовательное выполнение задач в целом ведет к увеличению времени, затрачиваемого на их выполнение.

Если раньше многофункциональность была свойственна только выдающимся людям, то в сегодняшнем цифровом мире она стала обычным явлением, с которым так или иначе сталкивается каждый человек. Эта способность обусловлена современным образом жизни человека и, согласно эмпирическим исследованиям, может быть развита при помощи тренировок, например, вербальной памяти. Тем не менее суть многозадачности скорее не в одновременном выполнении нескольких задач, а в способности к рациональному распределению познавательных ресурсов. Другими словами, «эффективный многозадачник» - это человек, который последовательно выполняет поставленные задачи, планирует, правильно распределяет внимание и действует исходя из текущей ситуации, а не тот, кто делает одновременно несколько дел.

Несмотря на весь интерес к данному феномену, на сегодняшний день многозадачность не относится к числу хорошо изученных явлений. Актуальным следует признать подход к пониманию психологических основ данного феномена с позиций предложенной Д. Канеманом [5] ресурсной теории внимания. 


\section{2. Ресурсная модель внимания Д. Канемана как подход к пониманию многозадачности}

При изучении феномена многозадачности большая роль отводится процессам памяти и внимания. При анализе проблемы многозадачности все чаще опираются на ресурсную модель внимания Д. Канемана [5], несмотря на то, что она была разработана задолго до того, как проблема многозадачности приобрела сегодняшнюю актуальность.

Согласно ресурсному подходу к изучению внимания, когнитивные ресурсы, задействованные для решения одной задачи, не могут использоваться для решения других задач. Для решения задачи необходимо тем больше ресурсов, чем сложнее ее характер. Следовательно, чем больше ресурсов затрачивается на решение одной задачи, тем меньше ресурсов остается на решение других задач: тем больше ошибок будет допущено при решении других задач [11].

Д. Канеман рассматривает внимание как внутреннее усилие, связанное с решением задач [5]. В рамках этой теории он попытался учесть два аспекта внимания: ресурсный (умственное усилие) и селективный (избирательная направленность умственного усилия на определенный вид деятельности). Согласно данной теории:

- имеется центральный предел возможности выполнения когнитивной работы, который можно представить в виде ограниченного количества ресурсов внимания, или «умственного усилия».

- имеется возможность управлять распределением ресурсов внимания между несколькими задачами.

- успешность выполнения одновременного выполнения нескольких задач зависит от их сложности и от запросов к ограниченным ресурсам внимания со стороны каждой задачи.

- хотя число доступных ресурсов внимания постоянно, оно может незначительно изменяться в зависимости от внешних факторов и событий, а также от функционального состояния человека.

В ресурсной модели внимания Д. Канемана центральным блоком является механизм распределения ресурсов на выполнение различных деятельностей. Этот блок выполняет две функции: во-первых, выбирает те структуры и процессы переработки, на которые будут выделены ресурсы, во-вторых, дозирует ресурсы, другими словами, определяет, сколько именно ресурсов необходимо выделить для решения той или иной задачи. Решения этим механизмом принимаются в зависимости как от постоянных предпочтений, так и от ситуативных обстоятельств [2;11].

Дополнительно к центральному блоку, модель включает блоки доступных ресурсов внимания (зависят от общей активации организма, даже не связанной с познавательными задачами), действующих интенций (они обеспечивают произвольность обращения внимания на объекты, события или мысли в зависимости от целей и установок), поведенческих форм активности (так, для сенсорной переработки воздействующих на человека стимулов ресурсы внимания не нужны, но они необходимы для более глубокой переработки информации), блок оценки требований к ресурсам внимания (когда суммарный запрос на ресурсы внимания превышает допустимый объем, обеспечение активностей ресурсами прекращается, однако если задача усложняется, количество отведенных ресурсов и активация могут возрасти), а также блок постоянных детерминант (способствует незамедлительному выделению ресурсов внимания для переработки информации о внезапно появляющихся или движущихся объектах, для реагирования на свое имя и другие жизненно значимые стимулы).

Данная модель может быть рассмотрена с позиций многозадачности, поскольку обладает рядом важных моментов [11]. Первый момент заключается в гибкости распределения ресурсов внимания и изменчивости характера этого распределения при возникновении новых задач. Уделяя внимание решению одной задачи, при возникновении 
новых задач мы можем достаточно быстро переключаться между ними. При этом скорость переключений будет зависеть от количества и сложности выполняемых задач. При этом речь идет именно о переключении между задачами, а не об одновременном их выполнении.

Второй момент заключается в конкуренции за ограниченные ресурсы внимания одновременно возникших задач. Такая конкуренция создает помехи в решении всех текущих задач. При этом увеличение сложности задач может привести к истощению ресурсов.

Третий момент заключается в необходимости использования всех ресурсов внимания при решении сложной умственной задачи. При параллельном возникновении другой, даже несложной, задачи основное внимание направляется на нее, что приводит к появлению ошибок и снижению эффективности всей деятельности [10].

Кроме того, важным положением теории Д. Канемана является идея об индивидуальных различиях в ограниченности когнитивных ресурсов: этот тезис подтвержден большим количеством проведенных в психологии познания исследований.

Итак, феномен многозадачности затруднительно рассматривать отдельно от процессов внимания. Несмотря на то, что ресурсная модель Д. Канемана была создана в 1970-х гг., она способна прояснить конкретные аспекты феномена многозадачности, являющегося на сегодняшний день высокоактуальной проблемой.

\section{2. Постановка задачи исследования и процедура исследования проблематики многозадачности в условиях пребывания в виртуальной среде}

В данной работе проблематика многозадачности поднимается в контексте практического применения системы виртуальной реальности. Данный подход может считаться логичным: применение виртуальных систем в психологии представляет собой заметный и закономерный тренд $[1 ; 3 ; 4 ; 7 ; 8 ; 16]$. Помимо того обстоятельства, что поведение в условиях пребывания в виртуальной реальности может во многих случаях считаться многозадачным, применение подобных систем в качестве методического приема психологического исследования обладает ценным качеством: процедура исследования тождественна для каждого испытуемого, поэтому все эмпирические результаты, полученные в достаточно сложном и потому трудноповторимом в обычных условиях социальном окружении, допустимо сравнивать между собой.

С учетом данного обстоятельства, разработано и осуществлено исследование феномена многозадачности в условиях применения системы виртуальной реальности. Успешность деятельности испытуемых оценивалась как количество и качество допущенных ошибок в постепенно усложняющихся сериях эмпирического исследования. Процедура исследования была одинакова для каждого испытуемого, все они работали в шлеме виртуальной реальности, для них проецировалось одинаковое для всех изображение.

Исследование посвящено изучению особенностей влияния фоновой задачи наблюдения за поведением аватаров, составляющих виртуальную аудиторию в виде учебного класса, - при решении основной задачи, а именно выступлении перед такой аудиторией с повторением слышимого в наушниках текста, т.е. аудирования. При подготовке исследования были подобраны аудиозаписи шести текстов, продолжительность каждой - около двух минут. Жанр текстов - художественный (три текста) и научно-популярный (три текста). Все тексты записаны женским голосом, среднего темпа говорения, с паузами. Тексты подаются в наушники участнику исследования, находящемуся в комнате виртуальной реальности (CAVE) перед виртуальным классом. Виртуальный класс представляет собой аудиовизуальную трехмерную среду с видом от первого лица и изображает (см. Рис. 1) школьный класс: три 
ряда по четыре парты, за каждой из парт сидит один аватар-ученик (шесть мальчиков и шесть девочек).

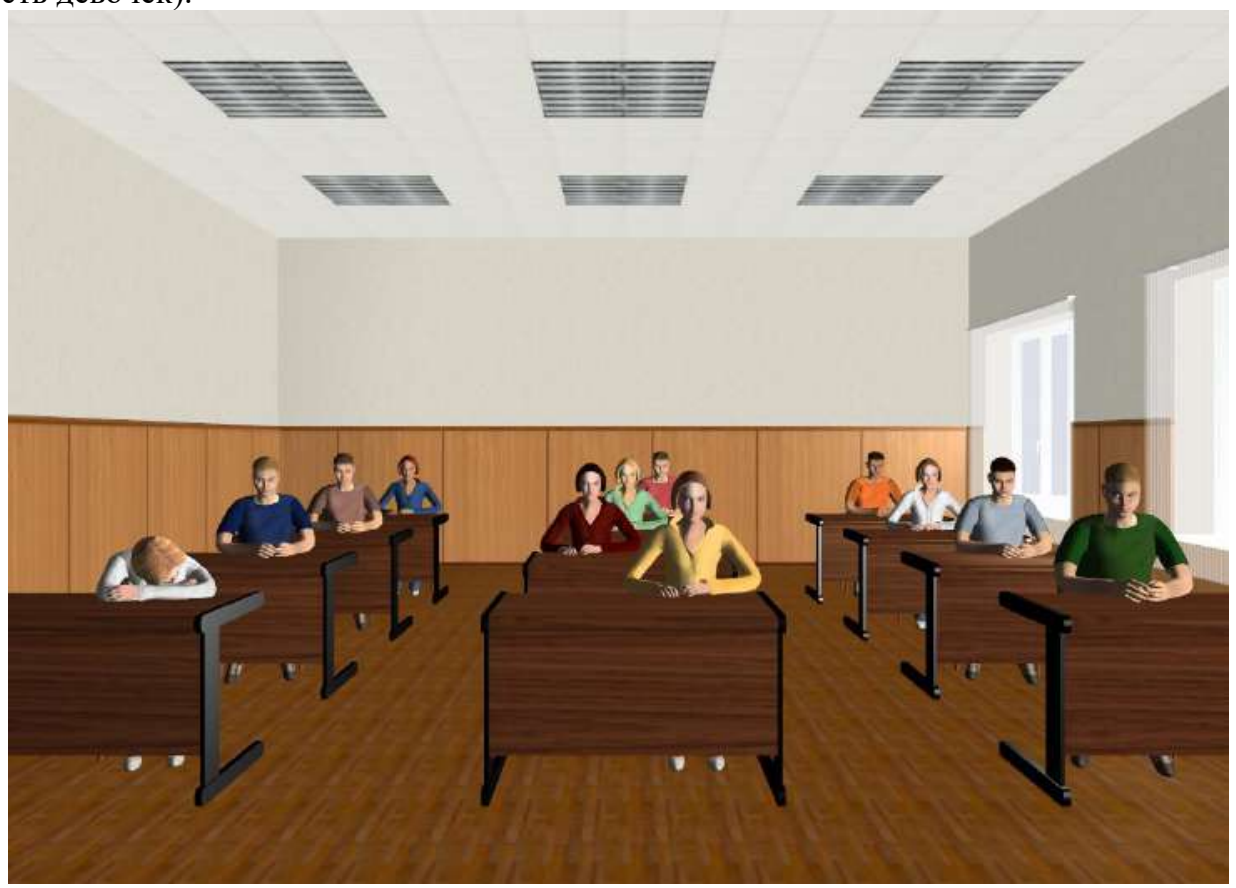

Рис. 1. Изображение виртуального класса

Исследование посвящено изучению особенностей влияния фоновой задачи наблюдения за поведением аватаров, составляющих виртуальную аудиторию в виде учебного класса, - при решении основной задачи, а именно выступлении перед такой аудиторией с повторением слышимого в наушниках текста, т.е. аудирования. При подготовке исследования были подобраны аудиозаписи шести текстов, продолжительность каждой - около двух минут. Жанр текстов - художественный (три текста) и научно-популярный (три текста). Все тексты записаны женским голосом, среднего темпа говорения, с паузами. Тексты подаются в наушники участнику исследования, находящемуся в комнате виртуальной реальности (CAVE) перед виртуальным классом. Виртуальный класс представляет собой аудиовизуальную трехмерную среду с видом от первого лица. Среда представляет собой школьный класс: три ряда по четыре парты, за каждой из парт сидит один аватар-ученик (шесть мальчиков и шесть девочек).

Испытуемым предстояло аудирование в условиях шести сценариев, в каждом из сценариев одинаковое количество «преимущественно внимательных» и «преимущественно невнимательных» учеников. Характеристика «внимательность ученика» задается набором поведенческих реакций, характеризующих каждого ученика. Реакции персонажей ограничены набором анимаций:

- смотрит на оратора;

- смотрит по сторонам;

- покашливает;

- копается в телефоне;

- выключает телефон при раздавшемся звонке;

- спит, положив голову на руки;

- разговаривает по телефону;

- кивает головой; 
- аплодирует;

- играет с телефоном.

Таким образом, были запрограммированы и потому не претерпевали каких-либо изменений реакции каждого ученика в течение шести двухминутных исследовательских серий. Количество невнимательных учеников в одном ряду - не более трех человек, на каждом ряду был предусмотрен хотя бы один невнимательный ученик.

К решению фоновой задачи подталкивали задаваемые испытуемым вопросы. А именно, после окончания каждой серии испытуемым задавались вопросы (всем в одинаковом порядке) - например, о том, сколько было внимательных и невнимательных учеников, за каким столом в каком ряду они сидели. Вопросы усложнялись от первой к шестой серии, так что согласно предположению, испытуемые, ожидая вопросы относительно виртуальной аудитории, должны были частично направить ресурсы памяти и внимания на фиксацию поведения виртуальных учеников, не переставая при этом аудировать тексты.

После окончания каждой серии участникам исследования задавались вопросы (всем в одинаковом порядке):

- после первого текста задавался вопрос «Как вы думаете, внимательно ли Вас слушала аудитория?».

- после второго текста: «Кто слушал Вас внимательней - люди на передних партах или на задних?»

- после третьего текста: «Кто слушал Вас внимательней - люди на ряду у окна, среднем ряду или у двери?», «Как Вы это определили?».

- после четвертого текста: «Удалось ли Вам запомнить, сколько в аудитории было мужчин и женщин?», «Сколько примерно людей слушали Вас внимательно, а сколько отвлекались?», «Как Вы определили, кто слушал Вас внимательно, а кто отвлекался?». А также давалось задание отметить на схеме класса расположение мужчин и женщин.

- после пятого текста: «Можете ли Вы вспомнить, что делали ученики, которые слушали Вас невнимательно?». А также давалось задание отметить на схеме класса тех учеников, которые, по мнению участника исследования, слушали его наиболее внимательно.

- после шестого текста: «Можете ли Вы вспомнить, что делали ученики, которые слушали Вас невнимательно?», «Мешали ли Вам ученики, которые отвлекались, или же это Вам было безразлично?». А также давалось задание отметить на схеме класса тех учеников, которые, по мнению участника исследования, слушали его/ее наиболее невнимательно.

Аудирование плюс контроль аудитории создают условия для многозадачности. Предполагалось, что ожидание все более сложных вопросов после каждой серии скажется на количестве и качестве допущенных испытуемыми ошибок аудирования.

Гипотеза: фоновая задача - уделение внимания поведению аватаров в составе виртуальной аудитории - оказывает влияние на успешность реализации основной задачи, а именно аудирования.

Эмпирическая гипотеза: имеется взаимосвязь между успешностью фиксации и запоминания поведения аватаров в виртуальной аудитории и количеством ошибок при аудировании.

Были разработаны шкалы ошибок аудирования и ошибок, допущенных при оценке поведения виртуальной аудитории. Исследование проведено на выборке студентов ВУЗов Москвы, обучающихся различным специальностям. В исследовании приняли участие 50 человек в возрасте от 18 до 25 лет (средний возраст 22 года; 26 женщин и 24 мужчины). Сбор эмпирических данных проводился индивидуально с каждым участником исследования. Исследование проведено в комнате виртуальной реальности (CAVE) на факультете психологии МГУ. 
В исследовании приняли участие 50 студентов различных ВУЗов Москвы в возрасте от 18 до 25 лет (средний возраст 22 года; 26 женщин и 24 мужчины). Исследование проведено в комнате виртуальной реальности (CAVE) на факультете психологии МГУ.

\section{3. Результаты и анализ результатов}

Проведен подсчет количества данных участниками исследования правильных ответов на вопросы о поведении аватаров в виртуальной аудитории. Проведена также классификация ошибок в аудировании текстов и составлена подробная таблица с допущенными ошибками. Допущенные ошибки были разделены на следующие категории:

- пропуск слова;

- пропуск фразы;

- пропуск абзаца;

- неразборчивое произнесение слова;

- замена слова на близкое по значению;

- замена слова на созвучное;

- замена слова на случайное слово;

- замена падежа/числа/окончания слова;

- осмысленное добавление слова или последовательности слов;

- добавление слова или последовательности слов с нарушением смысла.

Любопытно что, в ходе обработки данных появилась необходимость выделить две дополнительные группы ситуационных «ошибок»:

- жалобы участника эксперимента;

- попытки взаимодействовать с виртуальной аудиторией.

Далее все ошибки аудирования были сгруппированы по конкретным текстам, и был проведен однофакторный дисперсионный анализ (ANOVA) для проверки возможности влияния семантического компонента на результаты аудирования. В результате были исключены из дальнейшего рассмотрения около 10 зафиксированных ошибок (из более чем 2500 зафиксированных ошибок).

После этого были сопоставлены (1) условные баллы за правильные ответы на вопросы про поведение аватаров и (2) ошибки в аудировании, отсортированные по тематике текстов; был проведен корреляционный анализ по Спирмену. В результате был выявлен ряд значимых корреляций, и положительных, и (в меньшем количестве) отрицательных. Полученные значимые корреляции следует приравнять к артефактам, поскольку они не складываются в отчетливую картину: например, зафиксирована корреляция между ошибками внимания, допущенными после аудирования текста № 2, и ошибками аудирования текста № 4; не обнаружилось корреляционных связей между ошибками в аудировании единственного из шести текстов и правильными ответами на вопросы о поведении аватаров). Могут быть отмечены разве что определенные тенденции. Так, некоторые участники исследования, как бы вживаясь в роль «учителя», делали замечания учащимся в виртуальной аудитории, если замечали неподобающее, на их взгляд, поведение. Как можно предположить, некоторые отрицательные корреляционные связи является следствием того, что участники исследования, заметив «невнимательного» аватара, начинают следить за его поведением и перестают обращать внимание на других учащихся в аудитории, в итоге они ошибаются при ответе даже на несложный вопрос (напр., о количестве учащихся мужского и женского пола в аудитории).

Далее зафиксированные ошибки аудирования текстов были разделены на две группы, исходя из интуитивно понятных соображений. Действительно, ведь не все искажения следует считать существенными ошибками: имеются ошибки, которые реально приводят к смысловым или грамматическим искажениям, а вот другие ошибки пусть формально изменяют текст, но сохраняют неизменным смысл высказывания. Состав двух таких групп 
ошибок представлен в Табл. 1. Каждая из двух указанных групп может быть представлена отдельным фактором. В Табл. 1 приведены пункты, имеющие значимые нагрузки по соответствующему фактору с указанием этих нагрузок. Корреляция между факторами равна 0.624 .

Таблица 1. Состав факторов-групп ошибок со значимыми факторными нагрузками

\begin{tabular}{|c|c|c|c|}
\hline \multicolumn{2}{|c|}{ Первая группа ошибок } & \multicolumn{2}{|c|}{ Вторая группа ошибок } \\
\hline Тип ошибки & $\begin{array}{c}\text { Факторная } \\
\text { нагрузка }\end{array}$ & Тип ошибки & $\begin{array}{c}\text { Факторная } \\
\text { нагрузка }\end{array}$ \\
\hline $\begin{array}{l}\text { Замена падежа, числа, } \\
\text { окончания слова }\end{array}$ & 0.690 & Не произнесено фраза & 0.634 \\
\hline Замена слова на созвучное & 0.557 & $\begin{array}{l}\text { Осмысленное добавление } \\
\text { слова, последовательности } \\
\text { слов }\end{array}$ & 0.398 \\
\hline Слово не произнесено & 0.428 & $\begin{array}{l}\text { Замена слова на близкое по } \\
\text { значению }\end{array}$ & 0.253 \\
\hline $\begin{array}{l}\text { Проглатывание } \\
\text { неразборчивость }\end{array}$ & 0.409 & & \\
\hline $\begin{array}{l}\text { Замена слова на случайное } \\
\text { слово }\end{array}$ & 0.303 & & \\
\hline
\end{tabular}

Правомерность подобного разделения на группы подтверждена с помощью конфирматорного факторного анализа (GFI fit index 0.919, Root Mean-square Residual (RMR) 0.083).

В качестве общего вывода следует отметить следующие моменты. Была реализована попытка исследовать возможные связи между действиями в двух модальностях. Применение системы виртуальной реальности обеспечило возможность поставить всех без исключения участников исследования в одинаковые условия. Существенно, что ни добросовестное аудирование, ни фоновое наблюдение за поведением аватаров не могут вестись «по очереди», т.е. превращая одновременно выполняемые деятельности в прерываемые, или переключаемые деятельности. Тем самым полученные данные касательно феномена многозадачности должны быть признаны достоверными.

Полученные данные свидетельствуют, что имеются определенные связи между количеством и качеством ошибок аудирования. Часть таких ошибок можно соотнести с тем фактом, что задача аудирования является малознакомой для современного человека. Одновременно следует отметить, что имеются некоторые связи между «ошибками внимания», т.е. ошибками наблюдения за меняющимся согласно зафиксированному алгоритму поведением аватаров, составляющих виртуальную аудиторию, перед которой выступают участники исследования. К наблюдению за поведением аватаров участников исследования толкают фиксированные вопросы, которые задавались участникам после окончания каждого этапа аудирования. При желании они могли вообще не интересоваться поведением аватаров, однако среди участников исследования такие случаи не встретились.

Самый важный вывод состоит в том, что не выявлены значимые связи и зависимости между параллельно выполняемыми деятельностями в двух сенсорных модальностях. Сформулированные гипотезы не подтвердились. Отсутствие значимых различий подтверждено посредством однофакторного дисперсионного анализа с повторными измерениями.

Участники исследования успешно, однако не безошибочно справились с предложенным им заданием. Тем самым погрешности в выполнении одной деятельности не оказывают влияния на погрешности в выполнении другой деятельности. Поскольку погрешности - свидетельство затруднений, то следует признать, что в предложенных условиях у участников исследования протекали одновременно две деятельности (в разных сенсорных модальностях), причем затруднения в выполнении одной деятельности 
не связаны значимым образом с затруднениями в выполнении другой деятельности. Подобная разновидность многозадачности не ассоциируется с принятыми в когнитивной психологии ресурсными представлениями, согласно которым имеются общие когнитивные ресурсы, которые распределяются для обеспечения одновременно реализуемых активностей. Полученный результат в определенной мере противоречит представлению об общем когнитивном ресурсе для параллельно выполняемых деятельностей.

Поскольку модель внимания, согласно концепции Д. Канемана, включает помимо резервуара ресурсов и механизма распределения ресурсов, еще и особенности видов деятельности, на которые распределен ресурс, то можно предполагать, что примененные в нашей работе виды деятельности обладают некоторой малоучтенной в когнитивной психологии спецификой. Следует также обратить серьезное внимание на особенности взаимодействия между собой метакогнитивных стратегий в условиях постановки задач в тех сенсорных модальностях, которые были задействованы в данной работе. Таким образом, полученные результаты способствуют постановке новых исследовательских задач.

Работа выполнена при поддержке Российского научного фонда, проект № 18-18-00365.

\section{Литература}

[1] Архитектура виртуальных миров / под ред. М.Б. Игнатьева, А.В. Никитина, А.Е. Войскунского; 2-е изд., перераб. и доп. СПб.: ГУАП, 2009. 287 с.

[2] Величковский Б.М. Когнитивная наука: Основы психологии познания: в 2 т. Т. 1. М.: Смысл; Академия, 2006. 448 с.

[3] Войскунский А.Е., Меньшикова Г.Я. О применении систем виртуальной реальности в психологии // Вестник Московского университета. Серия 14. Психология. № 1, 2008, c. 22-36.

[4] Зинченко Ю.П. Психология виртуальной реальности. М.: МГУ, 2011. 359 с.

[5] Канеман Д. Внимание и усилие. М.: Смысл, 2006. 287 с.

[6] Креншоу Д. Миф о многозадачности: к чему приводит стремление успеть все. М.: Эксмо, 2010. $80 \mathrm{c}$

[7] Селиванов В.В., Селиванов А.В. Виртуальная субъектность: за и против // Методологические и медико-психологические аспекты здорового образа жизни. Смоленск: Универсум, 2003. С. 40-50.

[8] Селиванов В.В., Селиванова Л.Н. Влияние работы в виртуальной реальности на познавательные процессы и личностные особенности субъекта // Психология когнитивных процессов. 2017. № 3. С. 64-76.

[9] Смолл Г., Ворган Г. Мозг онлайн: Человек в эпоху Интернета. М.: КоЛибри, 2011. $352 \mathrm{c}$.

[10]Солдатова Г.У., Трифонова А.В. Медиамногозадачность: стоит ли беспокоиться // Дети в информационном обществе. 2018. № 28. С. 26-37.

[11]Фаликман М.В. Общая психология // Учебник для студентов высших учебных заведений / под ред. Б.С. Братуся: в 7 т. М.: Академия, 2006. Т. 4. Внимание.

[12]Courage M.L., Bakhtiar A., Fitzpatrick C., et al. Growing up multitasking: The costs and benefits for cognitive development // Developmental Review. 2015. Vol. 35. P. 5-41.

[13]Edwards K.S., Shin M. Media multitasking and implicit learning // Attention, Perception, \& Psychophysics. 2017. Vol. 79(5). P. 1535-1549.

[14]Ie A., Haller C. S., Langer E. J., Courvoisier D. S., Mindful multitasking: The relationship between mindful flexibility and media multitasking // Computers in Human Behavior. 2012. Vol. 28(4). P. 1526-1532. doi: 10.1016/j.chb.2012.03.022. 
[15]Limtrakul N., Louthrenoo O., Narkpongphun A., et al. Media use and psychosocial adjustment in children and adolescents // Journal of paediatrics and child health. 2018. Vol. 54, № 3. P. 296-301.

[16]Logie R.H., Trawley S., Law A. Multitasking: multiple, domain-specific cognitive functions in a virtual environment // Memory and Cognition. 2011. Vol. 39(8). P. 1561-1574. doi:10.3758/s13421-011-0120-1.

[17]Lui K.F., Wong A.C. Does media multitasking always hurt? A positive correlation between multitasking and multisensory integration // Psychonomic Bulletin \& Review. 2012. №. 19(4). P. 647-653.

[18]Moisala, M., Salmela, V., Hietajärvi, L., et al. Media multitasking is associated with distractibility and increased prefrontal activity in adolescents and young adults // NeuroImage. 2016. № 134. P. 113-121.

[19]Ophir E., Nass C., Wagner A.D. Cognitive control in media multitaskers // Proceedings of the National Academy of Sciences. 2009. Vol. 106, №37. P. 15583-15587.

[20]Rideout V.J., Foehr U.G., Roberts D.F. Generation M2: Media in the Lives of 8- to 18-YearOlds Generation // Kaiser Family Foundation Study. 2010. P. 33-34.

[21]Weigel M., Heikkinen K. Developing minds with digital media: Habits of mind in the YouTube era // Cambridge, MA: Harvard University Press. 2007.

[22]Voorveld H.A.M, Segijn C.M., Ketelaar P.E., et al. Investigating the prevalence and predictors of media multitasking across countries // International Journal of Communication. 2014. Vol. 8. P. 2755-2777.

[23] Voorveld H.A.M., Van der Goot M. Age differences in media multitasking: A diary study // Journal of Broadcasting \& Electronic Media. 2013. №. 57(3). P. 392-408.

[24]Yang T., Xie W., Chen C., et al. The development of multitasking in children aged 7-12 years: Evidence from cross-sectional and longitudinal data // Journal of experimental child psychology. 2017. №. 161. P. 63-80.

[25]Yang X., Zhu L. Predictors of media multitasking in Chinese adolescents // International Journal of Psychology. 2016. Vol. 51, № 6. P. 430-438.

[26]Zhang Y., Mao M., Rau P.-L. P., Choe P., Wang L.B.F. Exploring factors influencing multitasking interaction with multiple smart devices. Computers in Human Behavior. 2013. Vol. 29(6). P. 2579-2588. doi: 10.1016/j.chb.2013.06.042.

\title{
Multitasking in a Virtual Environment: Empirical Study
}

\author{
A.E. Voiskounsky, E.M. Khokhlova, O.V. Mitina, E.A. Dorokhov
}

\section{Moscow Lomonosov State University}

Multitasking issues, i.e. performing several different types of activity at once, has gained considerable interest both in the scientific community and among the broad masses of nonspecialists. This interest is due to a change in the rhythm of life in the modern time and, in particular, to the rapid development of electronic digital devices, which are increasingly mediating activities performed by people. To a large extent, interest is due to numerous observations, according to which representatives of youth generations not only do not experience problems in simultaneously performing different actions, but they also clearly prefer this style of life activity. The purpose of the work is to identify the specifics of the phenomenon of multitasking in terms of applying a virtual reality system. The study was conducted in a virtual reality room (CAVE) at the faculty of psychology at Moscow State University. The assignment for the subjects consisted of repeating oral texts while watching a virtual audience (a classroom with 12 avatar students) and simultaneously observing the behavior of the virtual avatars that make up the audience. Avatars had a fixed freedom of behavior, while their behavior was 
programmed and was the same for all study participants. The task for the subjects (50 students from various universities in Moscow) was gradually becoming more complicated, the degree of complexity can be judged by the number and quality of errors made by the participants in listening and in monitoring the audience. The study showed that difficulties in simultaneously implemented activities do not significantly affect each other.

Keywords: Multitasking, mediamultitasking, virtual reality, attention, resource model, avatar, repeating

Reference for citation: Voiskounsky A.E., Khokhlova E.M., Mitina O.V., Dorokhov E.A. Multitasking in a virtual environment: empirical study // Information Society: Education, Science, Culture and Technologies of the Future. Vol. 4 (Proceedings of the XXII International JointScientificConference «Internet and Modern Society», IMS-2020, St. Petersburg, June 17-20, 2020). - St. Petersburg: ITMO University, 2020. P. 129 - 142. DOI: $10.17586 / 2587-8557-2020-4-129-142$

\section{Reference}

[1] Arhitektura virtual'nyh mirov / pod red. M.B. Ignat'eva, A.V. Nikitina, A.E. Vo skunskogo; 2-e izd., pererab. i dop. SPb.: GUAP, 2009. 287 s. (in Russian).

[2] Velichkovskij B.M. Kognitivnaya nauka: Osnovy psihologii poznaniya: v 2 t. T. 1. M.: Smysl; Akademiya, 2006. 448 s. (in Russian).

[3] Vojskunskij A.E., Men'shikova G.YA. O primenenii sistem virtual'noj real'nosti v psihologii // Vestnik Moskovskogo universiteta. Seriya 14. Psihologiya. № 1, 2008, s. 22-36. (in Russian).

[4] Zinchenko YU.P. Psihologiya virtual'noj real'nosti. M.: MGU, 2011. 359 s. (in Russian).

[5] Kaneman D. Vnimanie i usilie. M.: Smysl, 2006. 287 s. (in Russian).

[6] Krenshou D. Mif o mnogozadachnosti: k chemu privodit stremlenie uspet' vse. M.: Eksmo, 2010. 80 s. (in Russian).

[7] Selivanov V.V., Selivanov A.V. Virtual'naya sub"ektnost': za i protiv // Metodologicheskie i mediko-psihologicheskie aspekty zdorovogo obraza zhizni. Smolensk: Universum, 2003. S. 40-50. (in Russian).

[8] Selivanov V.V., Selivanova L.N. Vliyanie raboty v virtual'noj real'nosti na poznavatel'nye processy i lichnostnye osobennosti sub"ekta // Psihologiya kognitivnyh processov. 2017. № 3. S. 64-76. (in Russian).

[9] Smoll G., Vorgan G. Mozg onlajn: CHelovek v epohu Interneta. M.: KoLibri, 2011. 352 s.

[10]Soldatova G.U., Trifonova A.V. Mediamnogozadachnost': stoit li bespokoit'sya // Deti v informacionnom obshchestve. 2018. № 28. S. 26-37. (in Russian).

[11]Falikman M.V. Obshchaya psihologiya // Uchebnik dlya studentov vysshih uchebnyh zavedenij / pod red. B.S. Bratusya: v 7 t. M.: Akademiya, 2006. T. 4. Vnimanie. (in Russian).

[12]Courage M.L., Bakhtiar A., Fitzpatrick C., et al. Growing up multitasking: The costs and benefits for cognitive development // Developmental Review. 2015. Vol. 35. P. 5-41.

[13]Edwards K.S., Shin M. Media multitasking and implicit learning // Attention, Perception, \& Psychophysics. 2017. Vol. 79(5). P. 1535-1549.

[14]Ie A., Haller C. S., Langer E. J., Courvoisier D. S., Mindful multitasking: The relationship between mindful flexibility and media multitasking // Computers in Human Behavior. 2012. Vol. 28(4). P. 1526-1532. doi: 10.1016/j.chb.2012.03.022.

[15]Limtrakul N., Louthrenoo O., Narkpongphun A., et al. Media use and psychosocial adjustment in children and adolescents // Journal of paediatrics and child health. 2018. Vol. 54, № 3. P. 296-301.

[16]Logie R.H., Trawley S., Law A. Multitasking: multiple, domain-specific cognitive functions in a virtual environment // Memory and Cognition. 2011. Vol. 39(8). P. 1561-1574. doi:10.3758/s13421-011-0120-1 
[17]Lui K.F., Wong A.C. Does media multitasking always hurt? A positive correlation between multitasking and multisensory integration // Psychonomic Bulletin \& Review. 2012. №. 19(4). P. 647-653.

[18]Moisala, M., Salmela, V., Hietajärvi, L., et al. Media multitasking is associated with distractibility and increased prefrontal activity in adolescents and young adults // NeuroImage. 2016. № 134. P. 113-121.

[19]Ophir E., Nass C., Wagner A.D. Cognitive control in media multitaskers // Proceedings of the National Academy of Sciences. 2009. Vol. 106, №37. P. 15583-15587.

[20]Rideout V.J., Foehr U.G., Roberts D.F. Generation M2: Media in the Lives of 8- to 18-YearOlds Generation // Kaiser Family Foundation Study. 2010. P. 33-34.

[21]Weigel M., Heikkinen K. Developing minds with digital media: Habits of mind in the YouTube era // Cambridge, MA: Harvard University Press. 2007.

[22]Voorveld H.A.M, Segijn C.M., Ketelaar P.E., et al. Investigating the prevalence and predictors of media multitasking across countries // International Journal of Communication. 2014. Vol. 8. P. 2755-2777.

[23]Voorveld H.A.M., Van der Goot M. Age differences in media multitasking: A diary study // Journal of Broadcasting \& Electronic Media. 2013. №. 57(3). P. 392-408.

[24]Yang T., Xie W., Chen C., et al. The development of multitasking in children aged 7-12 years: Evidence from cross-sectional and longitudinal data // Journal of experimental child psychology. 2017. №. 161. P. 63-80.

[25]Yang X., Zhu L. Predictors of media multitasking in Chinese adolescents // International Journal of Psychology. 2016. Vol. 51, № 6. P. 430-438.

[26]Zhang Y., Mao M., Rau P.-L. P., Choe P., Wang L.B.F. Exploring factors influencing multitasking interaction with multiple smart devices. Computers in Human Behavior. 2013. Vol. 29(6). P. 2579-2588. doi: 10.1016/j.chb.2013.06.042. 